\title{
Pricing and Hedging Path-Dependent Options Under the CEV Process
}

\author{
Dmitry Davydov • Vadim Linetsky \\ Equities Quantitative Strategies, UBS Warburg, 677 Washington Boulevard, \\ Stamford, Connecticut 06901 \\ Department of Industrial Engineering and Management Sciences, \\ McCormick School of Engineering and Applied Sciences, Northwestern University, \\ 2145 Sheridan Road, Evanston, Illinois 60208 \\ dmitry.davydov@ubsw.com •linetsky@iems.nwu.edu
}

$\mathrm{M}$ uch of the work on path-dependent options assumes that the underlying asset price follows geometric Brownian motion with constant volatility. This paper uses a more general assumption for the asset price process that provides a better fit to the empirical observations. We use the so-called constant elasticity of variance (CEV) diffusion model where the volatility is a function of the underlying asset price. We derive analytical formulae for the prices of important types of path-dependent options under this assumption. We demonstrate that the prices of options, which depend on extrema, such as barrier and lookback options, can be much more sensitive to the specification of the underlying price process than standard call and put options and show that a financial institution that uses the standard geometric Brownian motion assumption is exposed to significant pricing and hedging errors when dealing in path-dependent options.

(Path-Dependent Options; Barrier Options; Lookback Options; Diffusion Processes; CEV Model; Generalized Bessel Process; Radial Ornstein-Uhlenbeck Process)

\section{Introduction}

A standard option gives its owner the right to buy (or sell) some asset in the future for a fixed price. The fixed price is known as the strike price. Call options confer the right to buy the asset, while put options confer the right to sell the asset. Path-dependent options represent extensions of this concept. For example, a lookback call option confers the right to buy an asset at its minimum price over some time period. A barrier option resembles a standard option except that the payoff also depends on whether or not the asset price crosses a certain barrier level during the option's life. Lookback options and barrier options are two of the most popular types of path-dependent options.

Most of the academic literature on path-dependent options follow the lead set by Black and Scholes (1973) and assume that the underlying asset price follows geometric Brownian motion with constant volatility. This implies that the future asset prices are lognormaly distributed and leads to tractable analytical formulae. ${ }^{1}$ However, the evidence indicates that this distributional assumption is not rich enough to capture the empirical observations. If the true asset price process was geometric Brownian motion with constant volatility, then the Black-Scholes formula could be used to find out this volatility by equating the model price of a standard option to its market price. The volatility thus obtained is known as the implied

\footnotetext{
${ }^{1}$ Merton (1973) derives a closed-form pricing formula for downand-out call options. Rubinstein and Reiner (1991) extend Merton's result to other types of barrier options. Goldman et al. (1979), Goldman et al. (1979), and Conze and Vishwanathan (1991) provide closed-form pricing formulae for lookback options.
} 
volatility of the option. Empirically, we find that the implied volatilities computed from market prices of options with different strike prices are not constant but vary with strike price. This variation is observed across a wide range of markets and underlying assets and is known as the implied volatility smile or frown depending on its shape. The lognormal assumption with constant volatility does not capture this effect.

There are different ways of extending the basic model to incorporate this feature. The present paper uses the constant elasticity of variance (CEV) diffusion to model asset prices. This process, first introduced to finance by Cox (1975), is capable of reproducing the volatility smile observed in the empirical data. Our paper uses this model to examine the pricing and hedging of lookback and barrier options.

The contributions of the present paper are two-fold. First, we derive solutions for barrier and lookback option prices under the CEV process in closed form. More generally, we first derive closed-form expressions for barrier and lookback option prices under a general time-homogeneous, one-dimensional diffusion in terms of the two independent solutions of the stationary Black-Scholes differential equation with state-dependent volatility. Specializing to the CEV process, we then derive closed-form expressions for the stationary solutions and compute barrier and lookback option prices under the CEV process.

Second, we use the closed-form pricing formulae to carry out a comparative statics analysis. We demonstrate that barrier and lookback option prices and hedge ratios under the $\mathrm{CEV}$ process can deviate dramatically from the lognormal values. Therefore, substantial model risk exposure exists for financial institutions making markets in path-dependent options. Strikingly, we find that deltas of up-andout calls, down-and-out puts, double knock-out, and lookback options can have different signs under the $\mathrm{CEV}$ and lognormal specifications. In these cases, delta hedging with a misspecified model can produce worse results than no hedging at all. Finally, to further assess model risk inherent in writing these contracts, we carry out a dynamic hedging simulation experiment that quantifies the impact of model misspecification on the outcome of dynamic hedging strategies. Our results indicate that it is much more important to have an accurate model specification for pricing and hedging barrier and lookback options than for standard options.

This paper is organized as follows. Section 2 introduces barrier and lookback options. Section 3 focuses on general valuation results. We derive a closed-form expression for the Laplace transform of a barrier option price in time to expiration under a time-homogeneous, one-dimensional diffusion. The formula involves two independent solutions of the stationary Black-Scholes differential equation with state-dependent volatility. Similarly, closed-form formulae for lookback options are given in terms of stationary solutions. In $\S 4$, we specialize to the CEV diffusion and derive closed-form expressions for the stationary solutions in terms of Whittaker and Bessel functions. In §5, we compute barrier and lookback option prices and sensitivities, conduct a comparative statics analysis, and carry out a dynamic hedging experiment to quantify model risk due to model misspecification faced by financial institutions making markets in path-dependent options. Section 6 concludes the paper. Proofs are collected in Appendix A. Appendix B discusses the case of positive elasticity. Appendix $C$ contains explicit expressions for the integrals entering the barrier option pricing formulae. Appendix D discusses Laplace transform inversion.

\section{The Contracts: Barrier and Lookback Options}

Barrier options are probably the oldest path-dependent options. Snyder (1969) describes down-and-out stock options as "limited risk special options." Merton (1973) derives a closed-form pricing formula for down-and-out calls under the lognormal assumption. A down-and-out call is identical to a European ${ }^{2}$ call with the additional provision that the contract is canceled (knocked out) if the underlying asset price hits a prespecified lower barrier level. The contract may also specify a cash rebate to be received by the option holder if cancellation occurs. The rebate is received when the knock-out barrier is first reached.

\footnotetext{
${ }^{2}$ European options can only be exercised on the expiration date, while American options can be exercised on any business day through and including expiration.
} 
An up-and-out call is the same, except the contract is canceled when the underlying asset price first reaches a prespecified upper barrier level. A cash rebate may also be received when the barrier is first reached. Unlike down-and-out calls which are canceled outof-the-money (the lower barrier is placed below the strike price), up-and-out calls are canceled in-themoney (the upper barrier is placed above the strike). Generally, contracts that are canceled in-the-money are called reverse knock-out options.

Down-and-out and up-and-out puts are similar modifications of European put options. Knock-in options are complementary to the knock-out options: They pay off at expiration if and only if the underlying asset price does reach the prespecified barrier. The combination of otherwise identical in and out options is equivalent to the corresponding standard European option. Rubinstein and Reiner (1991) derive closed-form pricing formulae for all eight types of single-barrier options under the lognormal assumption.

Double-barrier (double knock-out) options are canceled when the underlying asset first reaches either the upper or the lower barrier. A rebate may also be received at that time. Different representations of the closed-form pricing formula for double barrier options under the lognormal assumption are obtained by Kunitomo and Ikeda (1992), Geman and Yor (1995), Pelsser (2000), and Schroder (2000).

A capped call is an up-and-out call with the cash rebate equal to the difference between the upper barrier (cap) and the strike price. It combines a European exercise feature and an automatic exercise feature. The automatic exercise is triggered when the index value first exceeds the cap (Broadie and Detemple 1995).

Barrier options are one of the most popular types of path-dependent options traded over-the-counter on stocks, stock indexes, currencies, commodities, and interest rates. Derman and Kani (1996) offer a detailed discussion of their investment, hedging, and trading applications. There are several reasons to use barrier options rather than standard options. First, barrier options may more closely match investor beliefs about the future behavior of the asset. By buying a barrier option, one can eliminate paying for those scenarios one feels are unlikely. Second, barrier option premiums are generally lower than those of standard options because an additional condition has to be met for the option holder to receive the payoff (e.g., the lower barrier not reached for down-and-out options). The premium reduction can be substantial, especially when volatility is high.

Lookback options are another important example of path-dependent options. Their payoff depends on the maximum or minimum underlying asset price attained during the option's life. A standard lookback call gives the option holder the right to buy at the lowest price recorded during the option's life. A standard lookback put gives the right to sell at the highest price recorded during the option's life. Lookbacks were first studied by Goldman, Sosin, and Gatto (1979) and Goldman, Sosin, and Shepp (1979) who derived closed-form pricing formulae under the lognormal assumption. In addition to standard lookback options, Conze and Vishwanathan (1991) introduce calls on maximum and puts on minimum. A call on maximum pays off the difference between the realized maximum price and some prespecified strike or zero, whichever is greater. A put on minimum pays off the difference between the strike and the realized minimum price or zero, whichever is greater. These options are called fixed-strike lookbacks. In contrast, the standard lookback options are also called floating-strike lookbacks, because the floating terminal underlying price $S_{T}$ serves as the strike price in standard lookback options.

\section{General Valuation Results}

We first develop some results for a general one-dimensional diffusion and later specialize to the CEV assumption. We take an equivalent martingale measure (risk-neutral probability measure) $\mathbf{Q}$ as given (Duffie 1996). Under $\mathbf{Q}$, we suppose that the asset price $\left\{S_{t}, t \geq 0\right\}$ is a time-homogeneous, nonnegative diffusion process solving the stochastic differential equation

$$
d S_{t}=\mu S_{t} d t+\sigma\left(S_{t}\right) S_{t} d B_{t}, \quad t \geq 0, \quad S_{0}=S>0,
$$

where $\left\{B_{t}, t \geq 0\right\}$ is a standard Brownian motion defined on a filtered probability space $\left(\Omega, \mathscr{F},\left\{\mathscr{F}_{t}\right\}_{t \geq 0}, \mathbf{Q}\right), \mu$ is a constant $(\mu=r-q$, where $r \geq 0$ and $q \geq 0$ are the constant risk-free interest 
rate and the constant dividend yield, respectively), and $\sigma=\sigma(S)$ is a given local volatility function, which is assumed continuous and strictly positive for all $S \in(0, \infty)$. We also assume that the local volatility function remains bounded as $S \rightarrow \infty$. This is sufficient to insure that infinity is a natural boundary ${ }^{3}$ for the diffusion (1). The boundary behavior at the origin depends on the growth behavior of $\sigma(S)$ as $S \rightarrow 0$. If $\sigma(S)$ remains bounded as $S \rightarrow 0$, then zero is a natural boundary. If $\sigma(S)$ grows as $S^{-p}$ with some $0<p \leq 1 / 2$ as $S \rightarrow 0$, then it is an exit boundary (bankruptcy). If $\sigma(S)$ grows as $S^{-p}$ with some $p>1 / 2$ as $S \rightarrow 0$, then zero is a regular boundary point, and we specify it as a killing boundary by adjoining a killing boundary condition (bankruptcy).

Throughout this paper $t$ denotes the running time variable. We assume that all options are written at time $t=0$ and expire at time $t=T>0$. Time remaining to expiration is denoted by $\tau=T-t$. Suppose the initial asset price is $S$ and the lower and upper barrier levels are $L$ and $U, L<S<U$. Define the first hitting time of the lower barrier $\mathscr{T}_{L}:=\inf \{t \geq$ $\left.0 ; S_{t}=L\right\}$, the first hitting time of the upper barrier $\mathscr{T}_{U}:=\inf \left\{t \geq 0 ; S_{t}=U\right\}$, and the first exit time from an interval between the two barriers $\mathscr{T}_{(L, U)}:=$ $\inf \left\{t \geq 0 ; S_{t} \notin(L, U)\right\}$ (by convention, the infimum of the empty set is infinity). Then a down-and-out call with strike price $K$ and no rebate has the payoff at expiration $\mathbf{1}_{\left\{\mathscr{J}_{L}>T\right\}}\left(S_{T}-K\right)^{+}$, where $\mathbf{1}_{\{A\}}$ is the indicator function of the event $A$, and $x^{+} \equiv \max \{x, 0\}$ is the positive part of $x$. The up-and-out call has the payoff $\mathbf{1}_{\left\{\Im_{U}>T\right\}}\left(S_{T}-K\right)^{+}$. The double-barrier call has the payoff $\mathbf{1}_{\left\{\sigma_{(L, U)}>T\right\}}\left(S_{T}-K\right)^{+}$. Rebates are fixed cash amounts paid at times $\theta=\mathscr{T}_{L}, \mathscr{T}_{U}$, or $\mathscr{T}_{(L, U)}$, given $\theta \leq T$.

Before attacking the problem of pricing finitely lived barrier options with rebates and expiration $T<\infty$, it is convenient to examine first three more primitive securities: perpetual claims that pay one dollar at times $\mathscr{T}_{L}, \mathscr{T}_{U}$, and $\mathscr{T}_{(L, U)}$, respectively, and have no set expiration date (see Ingersoll 1987, p. 371). The claim that pays one dollar at $\mathscr{T}_{(L, U)}$ can be decomposed into a combination of two additional

\footnotetext{
${ }^{3}$ The boundary classification of one-dimensional diffusions due to Feller is described in Karlin and Taylor (1981, Chapter 15) and Borodin and Salminen (1996, Chapter 2).
}

claims, the first claim paying one dollar at $\mathscr{T}_{L}$, given the lower barrier is reached first $\left(\mathscr{T}_{L}<\mathscr{T}_{U}\right)$, and the second claim paying one dollar at $\mathscr{T}_{U}$, given the upper barrier is reached first $\left(\mathscr{T}_{U}<\mathscr{T}_{L}\right)$.

Proposition 1. Suppose the risk-neutral asset price process is a diffusion (1) and the constant risk-free interest rate is $r>0$. Then the prices at time $t=0$ of the five perpetual claims described above are (the expectation $\mathbf{E}_{S}$ is with respect to the risk-neutral measure $\mathbf{Q}$ and the subscript $S$ indicates that the process (1) is starting at $S_{0}=S$ ):

- One dollar paid at $\mathscr{T}_{L}$ :

$$
\mathbf{E}_{S}\left[e^{-r T_{L}} \mathbf{1}_{\left\{\sigma_{L}<\infty\right\}}\right]=\frac{\phi_{r}(S)}{\phi_{r}(L)}, \quad S \geq L,
$$

- One dollar paid at $\mathscr{T}_{L}$, given $\mathscr{T}_{L}<\mathscr{T}_{U}$ :

$$
\mathbf{E}_{S}\left[e^{-r \mathscr{T}_{L}} \mathbf{1}_{\left\{\mathscr{T}_{L}<T_{U}\right\}}\right]=\frac{\Delta_{r}(S, U)}{\Delta_{r}(L, U)}, \quad L \leq S \leq U,
$$

- One dollar paid at $\mathscr{T}_{U}$ :

$$
\mathbf{E}_{S}\left[e^{-r \mathscr{T}_{u}} \mathbf{1}_{\left\{\mathscr{T}_{U}<\mathscr{T}_{0}\right\}}\right]=\frac{\psi_{r}(S)}{\psi_{r}(U)}, \quad S \leq U,
$$

- One dollar paid at $\mathscr{T}_{U}$, given $\mathscr{T}_{U}<\mathscr{T}_{L}$ :

$$
\mathbf{E}_{S}\left[e^{-r \mathscr{T}_{U}} \mathbf{1}_{\left\{\mathcal{J}_{U}<\mathscr{T}_{L}\right\}}\right]=\frac{\Delta_{r}(L, S)}{\Delta_{r}(L, U)}, \quad L \leq S \leq U,
$$

- One dollar paid at $\mathscr{T}_{(L, U)}$ :

$$
\mathbf{E}_{S}\left[e^{-r \mathscr{T}_{(L, U)}}\right]=\frac{\Delta_{r}(L, S)+\Delta_{r}(S, U)}{\Delta_{r}(L, U)}, \quad L \leq S \leq U,
$$

where (for any $0<A<B<\infty$ )

$$
\Delta_{r}(A, B):=\phi_{r}(A) \psi_{r}(B)-\psi_{r}(A) \phi_{r}(B) .
$$

The functions $\psi_{r}(S)$ and $\phi_{r}(S)$ can be characterized as the unique (up to a multiplicative constant) solutions of the ordinary differential equation $(O D E)^{4}$

$$
\frac{1}{2} \sigma^{2}(S) S^{2} \frac{d^{2} u}{d S^{2}}+\mu S \frac{d u}{d S}-r u=0, \quad S \in(0, \infty),
$$

first by demanding that $\psi_{r}(S)$ is increasing in $S$ and $\phi_{r}(S)$ is decreasing in $S$, and secondly, if the origin is a regular boundary point, posing a killing boundary condition:

$$
\psi_{r}(0+)=0 .
$$

\footnotetext{
${ }^{4}$ In our characterization of the functions $\psi_{r}$ and $\phi_{r}$, we follow Borodin and Salminen (1996, pp. 18-19). The subscript $r$ indicates the dependence on the risk-free rate that enters the ODE (8).
} 
The functions $\psi_{r}(S)$ and $\phi_{r}(S)$ have the following properties (Borodin and Salminen 1996, pp. 18-19). If zero is an exit boundary, then

$$
\psi_{r}(0+)=0, \quad \phi_{r}(0+)<+\infty .
$$

If zero is a natural boundary, then

$$
\psi_{r}(0+)=0, \quad \phi_{r}(0+)=+\infty .
$$

Because we assume that $\sigma(S)$ is bounded as $S \rightarrow \infty$, $+\infty$ is a natural boundary and

$$
\lim _{S \rightarrow \infty} \psi_{r}(S)=+\infty, \quad \lim _{S \rightarrow \infty} \phi_{r}(S)=0 .
$$

The functions $\psi_{r}(S)$ and $\phi_{r}(S)$ are called fundamental solutions of the ODE (8). They are linearly independent and all solutions can be expressed as their linear combinations. Moreover, the Wronskian $w_{r}$, defined by

$$
\phi_{r}(S) \frac{d \psi_{r}}{d S}(S)-\psi_{r}(S) \frac{d \phi_{r}}{d S}(S)=\mathfrak{s}(S) w_{r},
$$

is independent of $S$. Here $\mathfrak{s}(S)$ is the scale density of the diffusion (1)

$$
\mathfrak{s}(S)=\exp \left\{-\int^{S} \frac{2 \mu d x}{\sigma^{2}(x) x}\right\} .
$$

Proposition 1 expresses the prices of the five claims in terms of the two fundamental solutions of the stationary Black-Scholes differential equation with the local volatility function $\sigma(S)$ (note that the time derivative term is absent from Equation (8)). For geometric Brownian motion $S_{t}=S e^{\left(\mu-\sigma^{2} / 2\right) t+\sigma B_{t}}$ with constant volatility $\sigma$, the functional form of the fundamental solutions is (see Ingersoll 1987, p. 372, and Carr and Picron 1999):

$$
\begin{aligned}
\psi_{r}(S) & =S^{\gamma_{+}}, \quad \phi_{r}(S)=S^{\gamma_{-}}, \\
\gamma_{ \pm} & =-\gamma \pm \sqrt{\gamma^{2}+\frac{2 r}{\sigma^{2}}}, \\
\gamma & =\frac{\mu}{\sigma^{2}}-\frac{1}{2} .
\end{aligned}
$$

In $\S 4$ we present closed-form expressions for $\psi$ and $\phi$ for the CEV process.

To value cash rebates included in finitely lived knock-out option contracts with expiration $T<\infty$, we need to value claims that pay one dollar at times $\theta=$ $\mathscr{T}_{L}, \mathscr{T}_{U}$, or $\mathscr{T}_{L, U}$, given $\theta \leq T$. That is, we need to evaluate expectations of the form $\mathbf{E}_{\mathcal{S}}\left[\mathbf{1}_{\{\theta \leq T\}} e^{-r \theta}\right]$.
Proposition 2. For any $\lambda>0$, the Laplace transform of the rebate price in time to expiration ${ }^{5}$ is equal to $1 / \lambda$ times the price of the corresponding perpetual claim with the adjusted discount rate $r+\lambda$ :

$$
\int_{0}^{\infty} e^{-\lambda T} \mathbf{E}_{S}\left[\mathbf{1}_{\{\theta \leq T\}} e^{-r \theta}\right] d T=\frac{1}{\lambda} \mathbf{E}_{S}\left[e^{-(r+\lambda) \theta}\right] .
$$

Given the associated perpetual claim value (Proposition 1), the rebate price is found by inverting this Laplace transform.

Now we are ready to price terminal payoffs of finitely lived knock-out options. First, consider the more difficult case of double-barrier options. We need to evaluate the discounted expectation (in this paper we focus on calls; puts can be treated similarly)

$$
e^{-r T} \mathbf{E}_{S}\left[\mathbf{1}_{\left\{\mathscr{T}_{(L, U)}>T\right\}}\left(S_{T}-K\right)^{+}\right] .
$$

Proposition 3. Let $\mathfrak{s}(S)$ be the scale density (14) and $\mathfrak{m}(S)$ - the speed density ${ }^{6}$ of the diffusion (1)

$$
\mathfrak{m}(S)=\frac{2}{\sigma^{2}(S) S^{2} \mathfrak{s}(S)} .
$$

For $0<K \leq A<B<\infty$ and $\lambda>0$, define

$$
\begin{aligned}
& I_{\lambda}(K, A, B):=\int_{A}^{B}(Y-K) \psi_{\lambda}(Y) \mathfrak{m}(Y) d Y, \\
& J_{\lambda}(K, A, B):=\int_{A}^{B}(Y-K) \phi_{\lambda}(Y) \mathfrak{m}(Y) d Y,
\end{aligned}
$$

where $\psi_{\lambda}$ and $\phi_{\lambda}$ are the functions defined in Proposition 1 (with the risk-free rate $r$ replaced with $\lambda$ ). Then the Laplace

\footnotetext{
${ }^{5}$ It is sometimes easier to solve for the Laplace transform of an option price in time to expiration than for the option price itself. This Laplace transform (premultiplied by $\lambda$ ) can be interpreted as the price of an exponentially stopped option, i.e., an option expiring at a random independent exponential time (the first jump time of a Poisson process with intensity $\lambda$ and independent of the underlying asset price process). Geman and Yor (1993) use this idea to obtain closed-from solutions for arithmetic Asian options. Carr (1998) applies this idea to develop analytical approximations to value American options.

${ }^{6}$ See Karlin and Taylor (1981, p. 194) Karatzas and Shreve (1991, p. 343) and Borodin and Salminen (1996, p. 17) for discussions of scale and speed densities. Our definition of the speed density coincides with that of Karatzas and Shreve (1991) and Borodin and Salminen (1996) and differs from Karlin and Taylor (1981) who do not include two in the definition.
} 
transform of the expectation in Equation (17) in time to expiration is given by:

$$
\begin{gathered}
\int_{0}^{\infty} e^{-\lambda T} \mathbf{E}_{S}\left[\mathbf{1}_{\left\{\mathscr{T}_{(L, U)}>T\right\}}\left(S_{T}-K\right)^{+}\right] d T=\frac{1}{w_{\lambda} \Delta_{\lambda}(L, U)} \\
\times\left\{\begin{array}{cc}
\Delta_{\lambda}(L, S)\left[\psi_{\lambda}(U) J_{\lambda}(K, K, U)\right. \\
\left.-\phi_{\lambda}(U) I_{\lambda}(K, K, U)\right], & \text { if } S \leq K, \\
\Delta_{\lambda}(L, S)\left[\psi_{\lambda}(U) J_{\lambda}(K, S, U)\right. & \\
\left.-\phi_{\lambda}(U) I_{\lambda}(K, S, U)\right] & \\
+\Delta_{\lambda}(S, U)\left[\phi_{\lambda}(L) I_{\lambda}(K, K, S)\right. & \\
\left.-\psi_{\lambda}(L) J_{\lambda}(K, K, S)\right], & \text { if } S>K,
\end{array}\right.
\end{gathered}
$$

where $\Delta_{\lambda}(A, B)$ and $w_{\lambda}$ are defined in Equation (7) and Equation (13), respectively.

Then the double knock-out option price in Equation (17) is obtained by inverting the Laplace transform (21) and discounting at the risk-free rate. The single-barrier down-and-out (up-and-out) option prices are obtained by taking the limit $U \rightarrow \infty(L \rightarrow 0)$ in Equation (21) and using the boundary properties of the functions $\psi$ and $\phi$ given in Equations (9)-(12). In the interest of brevity we omit the resulting pricing formulae for single-barrier options. Finally, the capped call with the strike price $K$ and cap price $U$ is valued as a portfolio of an up-and-out call with the upper barrier $U$ and a claim that pays a cash rebate equal to the difference $(U-K)$ at the first hitting time $\mathscr{T}_{U}$, given $\mathscr{T}_{U} \leq T$.

We now turn to lookback options. To price lookback options we need distributions of the maximum and minimum prices.

LEMma 1. Let $M_{t}$ and $m_{t}$ be the maximum and minimum prices recorded to date $t, M_{t}=\max _{0 \leq u \leq t} S_{u}$ and $m_{t}=$ $\min _{0 \leq u \leq t} S_{u}$. Define the functions $F(y ; x, t):=\mathbf{Q}_{x}\left(m_{t} \leq y\right)$ and $G(y ; x, t):=\mathbf{Q}_{x}\left(M_{t} \geq y\right)$ (the probabilities are calculated with respect to the risk-neutral measure $\mathbf{Q}$ and the subscript $x$ indicates that the process is starting at $S_{0}=x$ ). Then for any $\lambda>0$

$$
\begin{array}{ll}
\int_{0}^{\infty} e^{-\lambda t} F(y ; x, t) d t=\frac{1}{\lambda} \frac{\phi_{\lambda}(x)}{\phi_{\lambda}(y)}, & 0<y \leq x, \\
\int_{0}^{\infty} e^{-\lambda t} G(y ; x, t) d t=\frac{1}{\lambda} \frac{\psi_{\lambda}(x)}{\psi_{\lambda}(y)}, & 0<x \leq y,
\end{array}
$$

where $\psi_{\lambda}$ and $\phi_{\lambda}$ are the functions defined in Proposition 1.
The probability distributions of the maximum and minimum are recovered by inverting the Laplace transforms. Lookback prices are expressed in terms of these probabilities.

Proposition 4. The prices of the standard lookback call, the standard lookback put, the call on maximum, and the put on minimum at some time $0 \leq t<T$ during the option's life are:

$$
\begin{aligned}
e^{-r \tau} \mathbf{E}_{t}\left[\left(S_{T}-m_{T}\right)^{+}\right]= & e^{-q \tau} S_{t}-e^{-r \tau} m_{t} \\
& +e^{-r \tau} \int_{0}^{m_{t}} F\left(y ; S_{t}, \tau\right) d y, \\
e^{-r \tau} \mathbf{E}_{t}\left[\left(M_{T}-S_{T}\right)^{+}\right]= & e^{-r \tau} M_{t}-e^{-q \tau} S_{t} \\
& +e^{-r \tau} \int_{M_{t}}^{\infty} G\left(y ; S_{t}, \tau\right) d y,
\end{aligned}
$$

$$
\begin{aligned}
e^{-r \tau} \mathbf{E}_{t}[ & \left.\left(M_{T}-K\right)^{+}\right] \\
= & \left\{\begin{array}{cc}
e^{-r \tau} \int_{K}^{\infty} G\left(y ; S_{t}, \tau\right) d y, & M_{t} \leq K, \\
e^{-r \tau} M_{t}-e^{-r \tau} K & \\
+e^{-r \tau} \int_{M_{t}}^{\infty} G\left(y ; S_{t}, \tau\right) d y, & M_{t}>K,
\end{array}\right. \\
e^{-r \tau} \mathbf{E}_{t}\left[\left(K-m_{T}\right)^{+}\right] & \left\{\begin{array}{cc}
e^{-r \tau} \int_{0}^{K} F\left(y ; S_{t}, \tau\right) d y, & m_{t} \geq K, \\
e^{-r \tau} K-e^{-r \tau} m_{t} & \\
+e^{-r \tau} \int_{0}^{m_{t}} F\left(y ; S_{t}, \tau\right) d y, & m_{t}<K,
\end{array}\right.
\end{aligned}
$$

where all contracts are initiated at time zero, $\mathbf{E}_{t}[\cdot] \equiv$ $\mathbf{E}\left[\cdot \mid \mathscr{F}_{t}\right], m_{t}$ and $M_{t}$ are the minimum and maximum prices recorded to date $t$ (known at time $t), S_{t}$ is the current underlying asset price at time $t$, and $\tau=T-t$ is the time remaining to expiration. 


\section{The CEV Process}

In this Section we specialize to the constant elasticity of variance (CEV) process of Cox $(1975)^{7}$

$$
d S_{t}=\mu S_{t} d t+\delta S_{t}^{\beta+1} d B_{t}, \quad t \geq 0, \quad S_{0}=S>0 .
$$

The CEV specification (28) nests the lognormal model of Black and Scholes (1973) and Merton (1973) $(\beta=0)$ and the absolute $(\beta=-1)$ and square-root $(\beta=-1 / 2)$ models of Cox and Ross (1976). For $\beta>0(\beta<0)$, local volatility $\sigma(S)=\delta S^{\beta}$ monotonically increases (decreases) as the asset price increases. The two model parameters $\beta$ and $\delta$ can be interpreted as the elasticity of the local volatility, $d \sigma / d S=\beta \sigma / S$, and the scale parameter fixing the initial instantaneous volatility at time $t=0, \sigma_{0}=\sigma\left(S_{0}\right)=\delta S_{0}^{\beta}$, respectively. Cox (1975) originally studied the case $\beta<0$. Emanuel and MacBeth (1982) extended his analysis to the case $\beta>0$. Cox originally restricted the elasticity parameter to the range $-1 \leq \beta \leq 0$. However, Reiner (1994) and Jackwerth and Rubinstein (1998) find that typical values of the CEV elasticity implicit in the S\&P 500 stock index option prices are strongly negative and are as low as $\beta=-4$. They term the corresponding model unrestricted CEV. The unrestricted CEV process is used to model the volatility smile effect in the equity index options market.

The CEV diffusion has the following boundary characterization. For $\beta<0$, infinity is a natural boundary. For $-1 / 2 \leq \beta<0$, the origin is an exit boundary. For $\beta<-1 / 2$, the origin is a regular boundary point and is specified as a killing boundary by adjoining a killing boundary condition. For $\beta=0$ (the lognormal model), both zero and infinity are natural boundaries. For $\beta>0$, the origin is a natural boundary and infinity is an entrance boundary. ${ }^{8}$

\footnotetext{
${ }^{7}$ Our parameter $\beta$ is defined as the elasticity of the local volatility function. Cox's elasticity parameter $\theta$ in $d S_{t}=\mu S_{t} d t+\delta S_{t}^{\theta / 2} d B_{t}$ is defined as the elasticity of the instantaneous variance of the asset price. The two parameters are related by: $\beta+1=\theta / 2$.

${ }^{8}$ In $\S 3$ we assumed that $\sigma(S)$ remains bounded as $S \rightarrow \infty$ and, consequently, $+\infty$ is a natural boundary. The CEV process with $\beta>0$ does not satisfy this assumption. This results in the nonexistence of an equivalent martingale measure for the CEV specification with $\beta>0$. To remedy the situation, one needs to regularize the process for large values. See Appendix $C$ for details.
}

The change of variable $z_{t}=(1 / \delta|\beta|) S_{t}^{-\beta}$ reduces the CEV process without drift $(\mu=0)$ to a standard Bessel process of order $1 /(2 \beta)$ (see Borodin and Salminen 1996, p. 66, and Revuz and Yor 1999, p. 439, for details on Bessel processes). The continuous part of the risk-neutral density of $S_{T}$, conditional on $S_{0}=S$, is obtained from the well known expression for transition density of the Bessel process (see Borodin and Salminen 1996, p. 115, and Revuz and Yor 1999, p. 446) and is given by $(\nu=1 /(2|\beta|))$

$$
\begin{aligned}
p_{0}\left(T ; S, S_{T}\right)= & \frac{S_{T}^{-2 \beta-\frac{3}{2}} S^{\frac{1}{2}}}{\delta^{2}|\beta| T} \exp \left(-\frac{S^{-2 \beta}+S_{T}^{-2 \beta}}{2 \delta^{2} \beta^{2} T}\right) \\
& \times I_{\nu}\left(\frac{S^{-\beta} S_{T}^{-\beta}}{\delta^{2} \beta^{2} T}\right),
\end{aligned}
$$

where $I_{\nu}$ is the modified Bessel function of the first kind of order $\nu$.

Using the result of Goldenberg (1991, p. 28), the CEV process with drift $\mu \neq 0$ is obtained from the process without drift via a scale and time change

$$
S_{t}^{(\mu)}=e^{\mu t} S_{\tau(t)}^{(0)}, \quad \tau(t)=\frac{1}{2 \mu \beta}\left(e^{2 \mu \beta t}-1\right) .
$$

Then the transition density with drift is obtained from the density (29) according to Goldenberg (1991, Proposition 2)

$$
p_{\mu}\left(T ; S, S_{T}\right)=e^{-\mu T} p_{0}\left(\tau(T) ; S, e^{-\mu T} S_{T}\right) \text {. }
$$

The density (31) was originally obtained by Cox (1975) for $\beta<0$ and by Emanuel and MacBeth (1982) for $\beta>0$ based on the result due to Feller (1951). For $\beta<0$, the risk-neutral probability of absorption at zero (bankruptcy), given $S_{0}=S$, is (Cox 1975)

$$
\mathbf{Q}_{S}\left(S_{T}=0\right)=G(\nu, \zeta / 2),
$$

where $G(\nu, x)$ is the complementary Gamma distribution function and $\zeta$ is defined in Equation (33) below.

The CEV density (31) can be expressed in terms of the noncentral chi-square density. Then the closedform CEV call option pricing formula can be expressed in terms of the complementary noncentral chi-square distribution function (Cox 1975 for $\beta<0$; Emanuel and MacBeth 1982 for $\beta>0$; Schroder 1989):

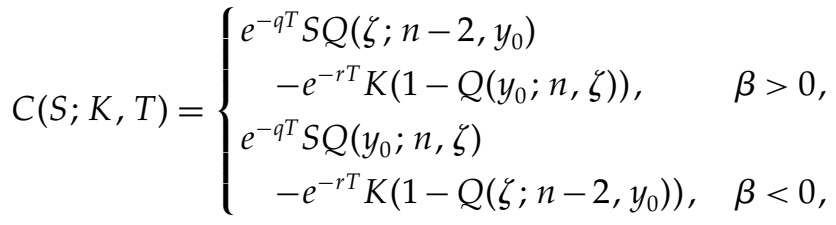


where

$$
\begin{aligned}
n & =2+\frac{1}{|\beta|}, \\
\zeta & =\frac{2 \mu S^{-2 \beta}}{\delta^{2} \beta\left(e^{2 \mu \beta T}-1\right)}, \\
y_{0} & =\frac{2 \mu K^{-2 \beta}}{\delta^{2} \beta\left(1-e^{-2 \mu \beta T}\right)},
\end{aligned}
$$

$K$ is the strike price of the call, and $Q(x ; u, v)$ is the complementary noncentral chi-square distribution function with $u$ degrees of freedom and the noncentrality parameter $v$. To compute the complementary noncentral chi-square distribution function we use the algorithm provided by Schroder (1989).

To value barrier and lookback options under the CEV process, we need to know the functional form of the fundamental solutions $\psi_{\lambda}$ and $\phi_{\lambda}$ similar to Equation (15) for geometric Brownian motion.

Proposition 5. Suppose $\beta \neq 0$ and $\lambda>0$. Introduce the following notation ${ }^{9}$

$$
\begin{gathered}
x:=\frac{|\mu|}{\delta^{2}|\beta|} S^{-2 \beta}, \quad z:=\frac{1}{\delta|\beta|} S^{-\beta}, \\
\epsilon=\operatorname{sign}(\mu \beta), \quad m:=\frac{1}{4|\beta|}, \\
k:=\epsilon\left(\frac{1}{2}+\frac{1}{4 \beta}\right)-\frac{\lambda}{2|\mu \beta|}, \quad \nu:=\frac{1}{2|\beta|} .
\end{gathered}
$$

The fundamental increasing $\left(\psi_{\lambda}\right)$ and decreasing $\left(\phi_{\lambda}\right)$ solutions of the CEV ODE

$$
\frac{1}{2} \delta^{2} S^{2 \beta+2} \frac{d^{2} u}{d S^{2}}+\mu S \frac{d u}{d S}-\lambda u=0, \quad S \in(0, \infty),
$$

are (up to multiplicative constants):

$$
\psi_{\lambda}(S)= \begin{cases}S^{\beta+\frac{1}{2}} e^{\frac{\epsilon}{2} x} M_{k, m}(x), & \beta<0, \mu \neq 0, \\ S^{\beta+\frac{1}{2}} e^{\frac{\epsilon}{2} x} W_{k, m}(x), & \beta>0, \mu \neq 0, \\ S^{\frac{1}{2}} I_{\nu}(\sqrt{2 \lambda} z), & \beta<0, \mu=0, \\ S^{\frac{1}{2}} K_{\nu}(\sqrt{2 \lambda} z), & \beta>0, \mu=0,\end{cases}
$$

${ }^{9}$ If $S_{t}$ follows the CEV diffusion (28), $x_{t}$ defined in Equation (34) follows a Feller (1951) diffusion: $d x_{t}=\left(b x_{t}+c\right) d x_{t}=\left(b x_{t}+c\right) d t-$ $\xi \sqrt{2 a x_{t}} d B_{t}$, where $a=2|\mu \beta|, b=-2 \mu \beta$ and $c=|\mu \beta|(2+1 / \beta)$. The process $z_{t}$ defined in Equation (34) follows a generalized Bessel diffusion: $d z_{t}=\left(d / z_{t}-\mu \beta z_{t}\right) d t-\xi d B_{t}$, where $d=(1+\beta) / 2 \beta, \xi=\operatorname{sign}(\beta)$. This process is also known as the radial Ornstein-Uhlenbeck process (Shiga and Watanabe 1973, Eie 1983, Going-Jaeschke and Yor 1999) and Rayleigh process (Giorno et al. 1986).

$$
\phi_{\lambda}(S)= \begin{cases}S^{\beta+\frac{1}{2}} e^{\frac{\epsilon}{2} x} W_{k, m}(x), & \beta<0, \mu \neq 0, \\ S^{\beta+\frac{1}{2}} e^{\frac{\epsilon}{2} x} M_{k, m}(x), & \beta>0, \mu \neq 0, \\ S^{\frac{1}{2}} K_{\nu}(\sqrt{2 \lambda} z), & \beta<0, \mu=0, \\ S^{\frac{1}{2}} I_{\nu}(\sqrt{2 \lambda} z), & \beta>0, \mu=0,\end{cases}
$$

where $M_{k, m}(x)$ and $W_{k, m}(x)$ are the Whittaker functions, ${ }^{10}$ and $I_{\nu}(x)$ and $K_{\nu}(x)$ are the modified Bessel functions. The Wronskian (13) of the functions $\psi_{\lambda}$ and $\phi_{\lambda}$ with respect to the scale density of the CEV diffusion,

$$
\mathfrak{s}(S)=\exp \left(\frac{\mu}{\delta^{2} \beta} S^{-2 \beta}\right),
$$

is

$$
w_{\lambda}= \begin{cases}\frac{2|\mu| \Gamma(2 m+1)}{\delta^{2} \Gamma\left(m-k+\frac{1}{2}\right)}, & \mu \neq 0, \\ |\beta|, & \mu=0,\end{cases}
$$

where $\Gamma(x)$ is the Euler Gamma function.

Equations (37) and (38) are the CEV counterparts of the solutions (15) for geometric Brownian motion. The CEV (double) barrier option pricing formula is obtained in the following steps. First, the functions (37) and (38) are substituted into Equations (19) and (20) and the integrals $I_{\lambda}$ and $J_{\lambda}$ are calculated. Fortunately, the integrals can be calculated in closed form and are given in Appendix C. Next, the integrals are substituted into Equation (21). Finally, to compute the price in Equation (17), the Laplace transform is inverted (see Appendix D). The rebates are priced by substituting (37) and (38) into Equations (2)-(6) and inverting the Laplace transform (16). The lookback options are priced by Equations (22)-(27). The outer integrals in $y$ in Equations (24)-(27) cannot be evaluated in closed form and must be computed numerically.

\section{Model Risk}

Armed with the pricing formulae, we are now ready to analyze the effect of $\beta$ on prices and hedge ratios of

\footnotetext{
${ }^{10}$ See Abramowitz and Stegun (1972, p. 505) and Slater (1960). The Whittaker functions are related to the confluent hypergeometric functions available in the Mathematica computer system. Whittaker functions also appear in similar contexts in Giorno et al. (1986) and Going-Jaeschke and Yor (1999) in connection with the calculation of Laplace transforms of first hitting times of generalized Bessel processes.
} 
Figure 1 European Call Prices and Black-Scholes Implied Volatilities as Functions of Strike $K$ Under CEV Processes with Elasticities $\beta=$ $0,-0.5,-1,-2,-3$, and -4 . Parameters: $S_{0}=100, \sigma_{0}=\sigma(100)=0.25, r=0.1, q=0, T=0.5$

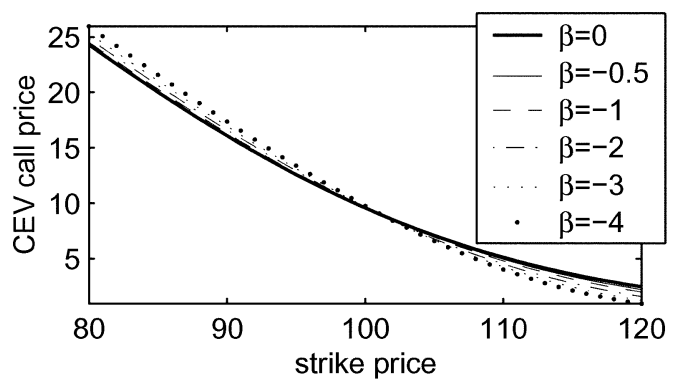

barrier and lookback options. To facilitate the comparison of our analytical results with numerical results already in the literature, we adopt the same choice of parameters as Boyle and Tian (1999). The initial asset price is $S_{0}=100$, the instantaneous volatility at this price level is $\sigma_{0}=25 \%$ per annum (i.e., the scale parameter $\delta$ is selected so that $\sigma_{0}=\sigma\left(S_{0}\right)=\delta S_{0}^{\beta}=0.25$ when $\left.S_{0}=100\right)$, the risk-free interest rate is $10 \%$ per annum $(r=0.1)$, the asset pays no dividends $(q=0)$, and all options have six months to expiration $(T=0.5)$. We employ six different values of $\beta$ to show its effect on option prices and hedge ratios ${ }^{11}$ : $\beta=0,-0.5,-1,-2,-3,-4$. The constant volatility case $(\beta=0)$ corresponds to the lognormal model. The negative elasticity values are characteristic of stock index options (Reiner 1994 and Jackwerth and Rubinstein 1998 find that the prices of S\&P 500 index options imply values of beta as low as $\beta=-4$ ). Following Boyle and Tian (1999), to ensure that option prices based on different values of $\beta$ are broadly comparable, the value of $\delta$ in each model is readjusted so that the initial instantaneous volatility is the same across different models. Let $\sigma_{0}$ be the instantaneous volatility for the lognormal model. Then the value of $\delta$ to be used for models with different $\beta$ values is adjusted to be $\delta=\sigma_{0} S_{0}^{-\beta}$.

\footnotetext{
${ }^{11}$ We focus our discussion on the case of negative $\beta$ because of its empirical importance for the stock index option market. Positive $\beta$ are characteristic of some commodity futures options with upward sloping implied volatility smiles.
}

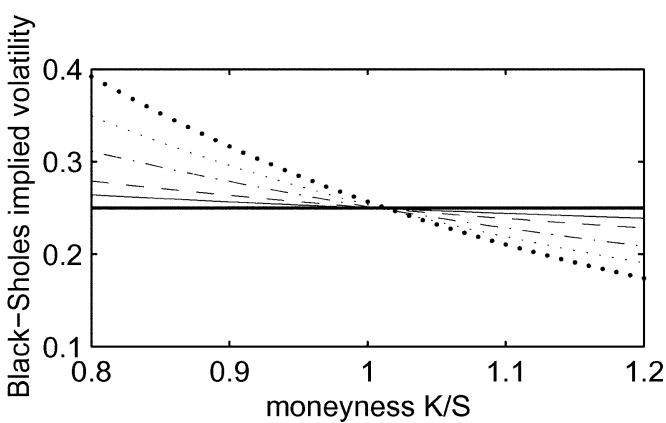

Figure 1 plots European call prices and Black-Scholes implied volatilities ${ }^{12}$ as functions of the strike price under the CEV processes with different values of $\beta$. Numerical values of prices and deltas are given in Table 1. Examination of the data reveals that in-themoney (out-of-the-money) calls under the CEV model with negative $\beta$ are worth more (less) than under the Black-Scholes model. The larger the absolute value of $\beta$, the greater the price difference between CEV option prices and Black-Scholes prices.

The Black-Scholes implied volatility of CEV calls exhibits a typical downward sloping volatility smile pattern (also called smirk, skew or frown), with higher implied volatilities corresponding to lower strikes (in-the-money calls) and lower implied volatilities corresponding to higher strikes (out-of-themoney calls). This is similar to the downward sloping implied volatility smile pattern observed in the S\&P 500 stock index options market after the crash of October 1987. Jackwerth and Rubinstein (1998) estimate the CEV model parameters $\beta$ and $\delta$ implicit in the six-month S\&P 500 option prices over the eight-year period from 1986 to 1994 using daily data. In their pre-crash sample, implicit values of $\beta$ are very close to zero, as predicted by the lognormal model. For several months after the October 1987 crash, the index option market underwent a transition with implied $\beta$ values steadily declining from zero into the -3 to -4 range. Since 1988, the S\&P 500 options market seems to settle into a stable regime

\footnotetext{
${ }^{12}$ Cox's formula (32) is first used to compute the CEV prices of options with different strikes. The Black-Scholes implied volatility is then calculated for each option.
} 
Table 1 CEV Barrier Options

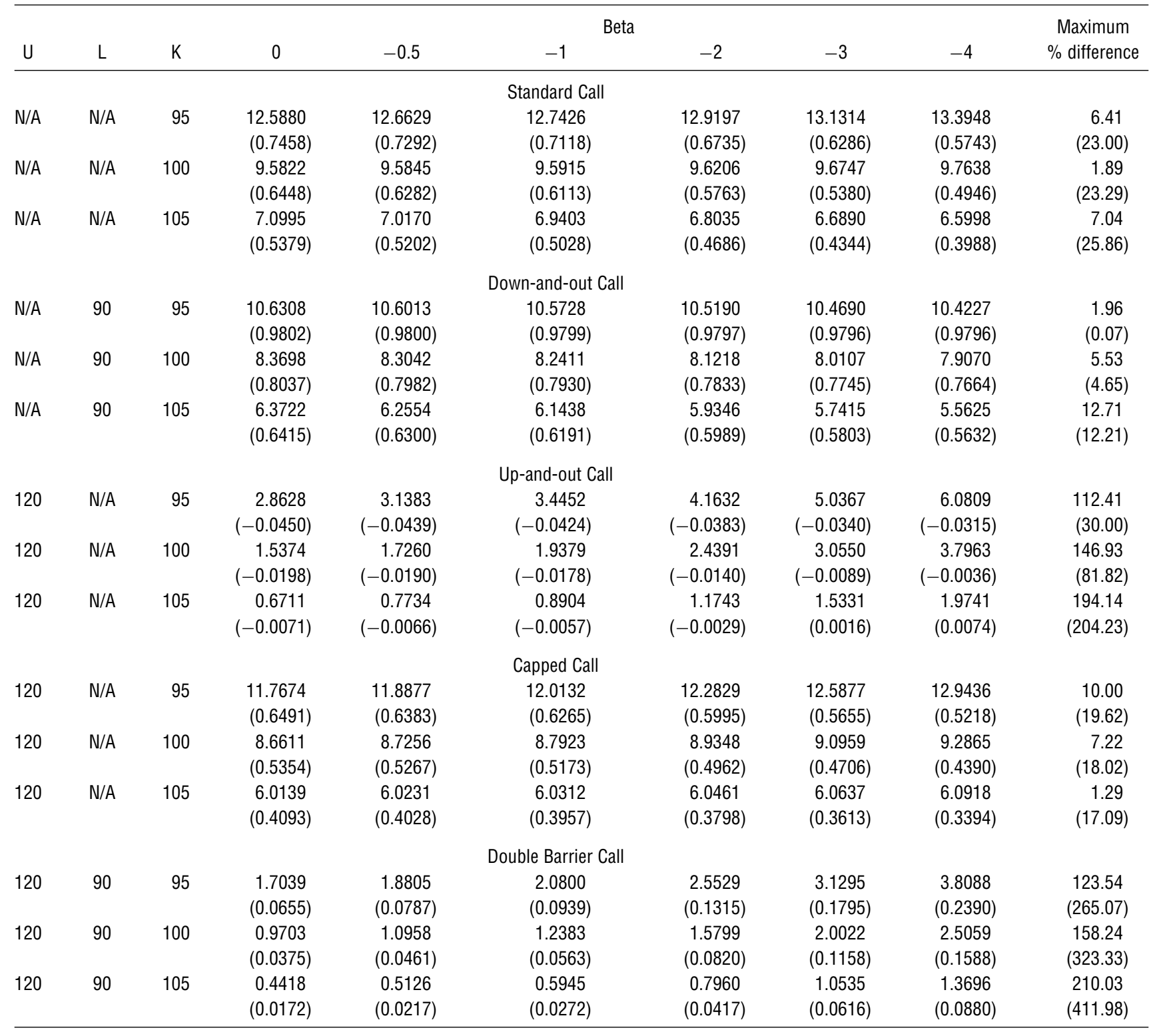

Note. Standard, down-and-out, up-and-out, capped, and double knock-out call prices and deltas under the CEV processes with elasticities $\beta=0,-0.5,-1,-2,-3$, and -4 . The value of delta is given in parentheses underneath the corresponding option price. The utmost right column gives the absolute value of the percentage difference between the Black-Scholes price (delta) and the CEV price (delta) with $\beta=-4$ relative to the Black-Scholes price (delta). The strike price $K$, upper barrier $U$ and lower barrier $L$ vary as indicated in the three left columns. Parameters used in the calculation: $S_{0}=100$, $\sigma_{0}=\sigma(100)=0.25, r=0.1, q=0, T=0.5$.

with $\beta$ estimates largerly confined to the -3 to -4 range. The mean of their daily estimates over the post-crash 1988-1994 period is close to -4 , with the mean at-the-money implied volatility of $17 \%$.
Overall, we note that the differences in call option prices and deltas with strikes close to at-the-money level are not all that significant under different choices of $\beta$. The differences increase for deep 
out-of-the-money and in-the-money options. As a result, errors resulting from misspecifying $\beta$ when pricing and hedging standard call options are relatively small, except for deep out- or in-the-money options.

In addition to standard call options, Table 1 reports prices and deltas of down-and-out, up-and-out, double knock-out and capped call options for different values of $\beta$. They are calculated using the analytical formulae derived in $\S 3$ and 4 . This table is the counterpart to Table 3 in Boyle and Tian (1999) who report prices of down-and-out, up-and-out and double knock-out calls calculated using their trinomial lattice with 1,000 time steps. Their trinomial results provide good approximation to our closedform solutions, generally agreeing with our option prices to three decimal places. ${ }^{13}$

The salient feature of the results is that the value of $\beta$ has a much greater impact on the prices of upand-out and double knock-out calls than on standard, capped and down-and-out calls. The maximum percentage differences in the CEV prices from the lognormal prices in our sample are $7.04 \%$ for standard calls, $10 \%$ for capped calls, $12.71 \%$ for down-andout calls, $194.14 \%$ for up-and-out calls and $210.03 \%$ for double knock-out calls. The maximum percentage differences in the CEV deltas from the lognormal deltas are $25.86 \%$ for standard calls, $19.62 \%$ for capped calls, $12.21 \%$ for down-and-out calls, $204.23 \%$ for up-and-out calls and $411.98 \%$ for double knockout calls. Thus, a misspecified value of $\beta$ may cause very large pricing and hedging errors for up-and-out and double knock-out calls. Strikingly, the up-and-out call delta can have different signs for different values of $\beta$ (e.g., the up-and-out call with the strike price of 105 in Table 1). In these cases, hedging with a misspecified model is worse than not hedging at all.

Given the fact that both the lognormal and the CEV models are calibrated so that the instantaneous volatility at the initial price level $S_{0}$ is the same across different models, these differences are purely the effect of the inverse relationship between volatility and the asset price level. The reason for this extreme

\footnotetext{
${ }^{13}$ Boyle and Tian (1999) report prices for $\beta$ restricted to the range $[-1,0]$ and do not report deltas.
}

sensitivity to the elasticity parameter is that up-andout and double knock-out calls (as well as down-andout and double knock-out puts) are canceled when the option is in-the-money. At the time of knockout at the upper barrier $U$, the call is in-the-money by the amount $U-K$ (\$20 in our example). A slight error in estimating knockout probability can result in large pricing and hedging errors as it is multiplied by the large dollar value.

Further, Figure 2 plots prices and deltas of standard, up-and-out, down-and-out, and double knockout calls as functions of the underlying asset price. The graphs confirm our observation that the prices of up-and-out and double knock-out calls are dramatically affected by the choice of $\beta$. At the same time, standard, capped and down-and-out calls are much more robust to the choice of $\beta$.

Table 2 illustrates the effect of $\beta$ on prices and deltas of lookback options. ${ }^{14}$ Maximum percentage differences in the CEV model prices and deltas from the lognormal ones are all greater for lookback options than for the corresponding standard options. Similar to up-and-out calls and down-and-out puts, deltas of standard lookback calls and puts can have different signs under the lognormal and CEV models (e.g., lognormal lookback call delta of 0.1563 vs. CEV delta of -0.5894 with $\beta=-4$ ). Thus, lookback options are also extremely sensitive to model misspecification.

To further assess model risk inherent in selling barrier options, we have carried out a dynamic hedging simulation experiment that quantifies the impact of model misspecification on the outcome of dynamic hedging strategies. Due to space limitations the results are not included in the published version. The description of the simulation experiment can be found in the working paper version of this article available on the web at $\langle$ http:// users.iems.nwu.edu/ linetsky/cev.pdf $\rangle$. The

\footnotetext{
${ }^{14}$ In contrast with barrier options, our exact lookback option prices reported in Table 2 are in disagreement with the trinomial results for lookbacks reported by Boyle and Tian (1999, in Tables 4 and 5). The apparent cause is a subtle error in their trinomial lattice algorithm for lookback options. See the correction: Boyle et al. (1999) "Lookback Options Under the CEV Process: A Correction" on the JFQA web site 〈http://depts.washington.edu/jfqa/〉 in "Notes, Comments, and Corrections."
} 
Figure 2 Standard Call, Down-and-Out (DAO) Call, Up-and-Out (UAO) Call, and Double Barrier Call Prices and Deltas As Functions of the Underlying Asset Price $S_{0}$ Under the CEV Processes with Elasticities $\beta=0,-0.5,-1,-2,-3$, and -4 . Parameters: $K=100, L=90, U=120, \sigma_{0}=$ $\sigma(100)=0.25, r=0.1, q=0, T=0.5$
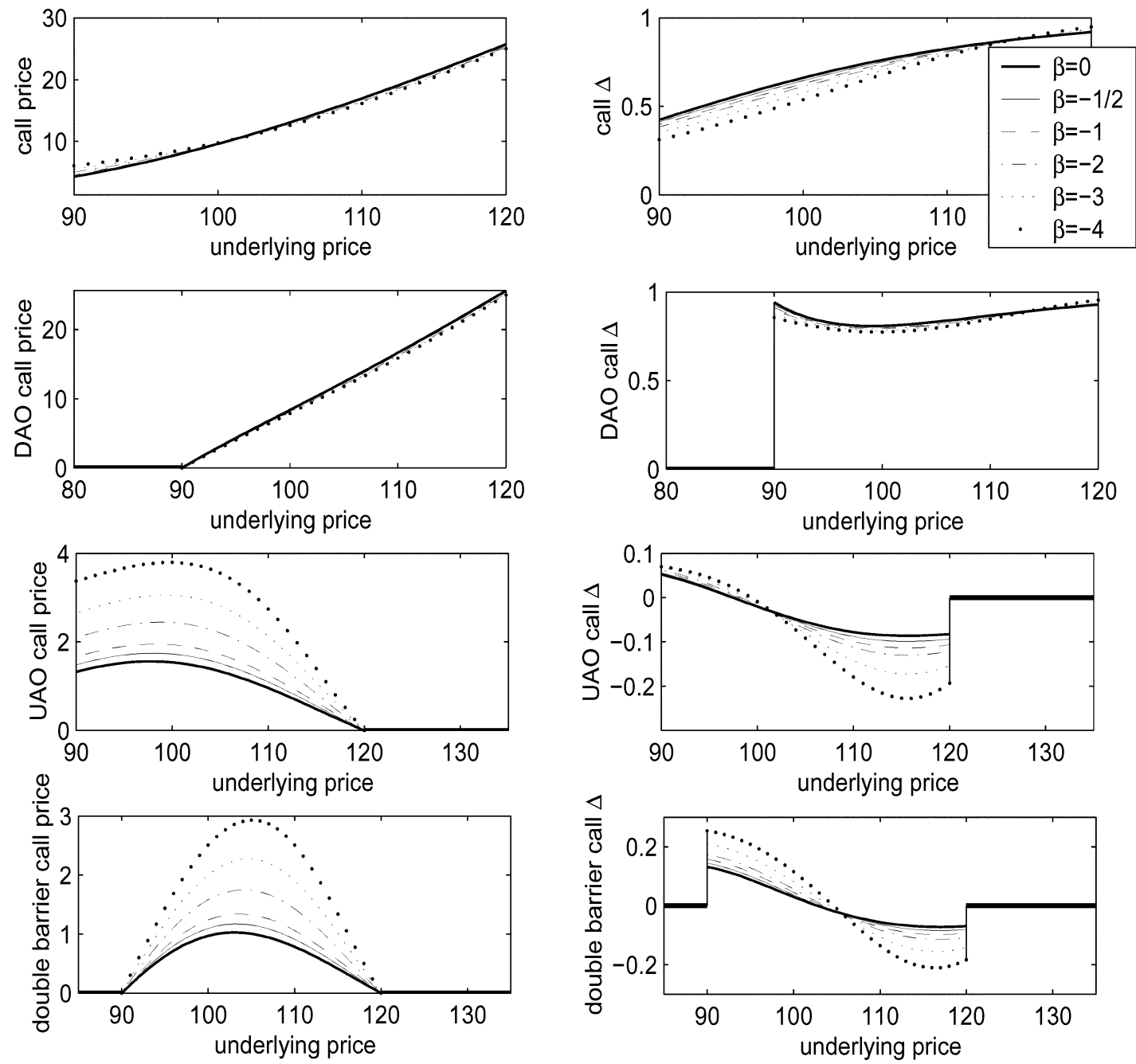

conclusion of the experiment is the observation that while the delta hedging strategy is fairly robust to model misspecification for standard, capped, and down-and-out calls, it is highly unstable for up-andout and double knock-out calls. For the latter contracts, it is possible to do worse by delta-hedging with a misspecified model than not hedging at all.

\section{Conclusion}

This paper studies the pricing and hedging of barrier and lookback options under the CEV process.

The CEV model with negative elasticity exhibits convex and monotonically decreasing implied volatility smiles similar to the smiles empirically observed in the stock index options market and, thus, allows us to study the effect of volatility smiles on pricing and hedging of path-dependent options.

The contributions of this study are two-fold. First, we derive pricing formulae for barrier and lookback options under the CEV process in closed form. Our analytical formulae allow fast and accurate calculation of prices and hedge ratios of barrier and lookback options under the CEV process on a PC. 


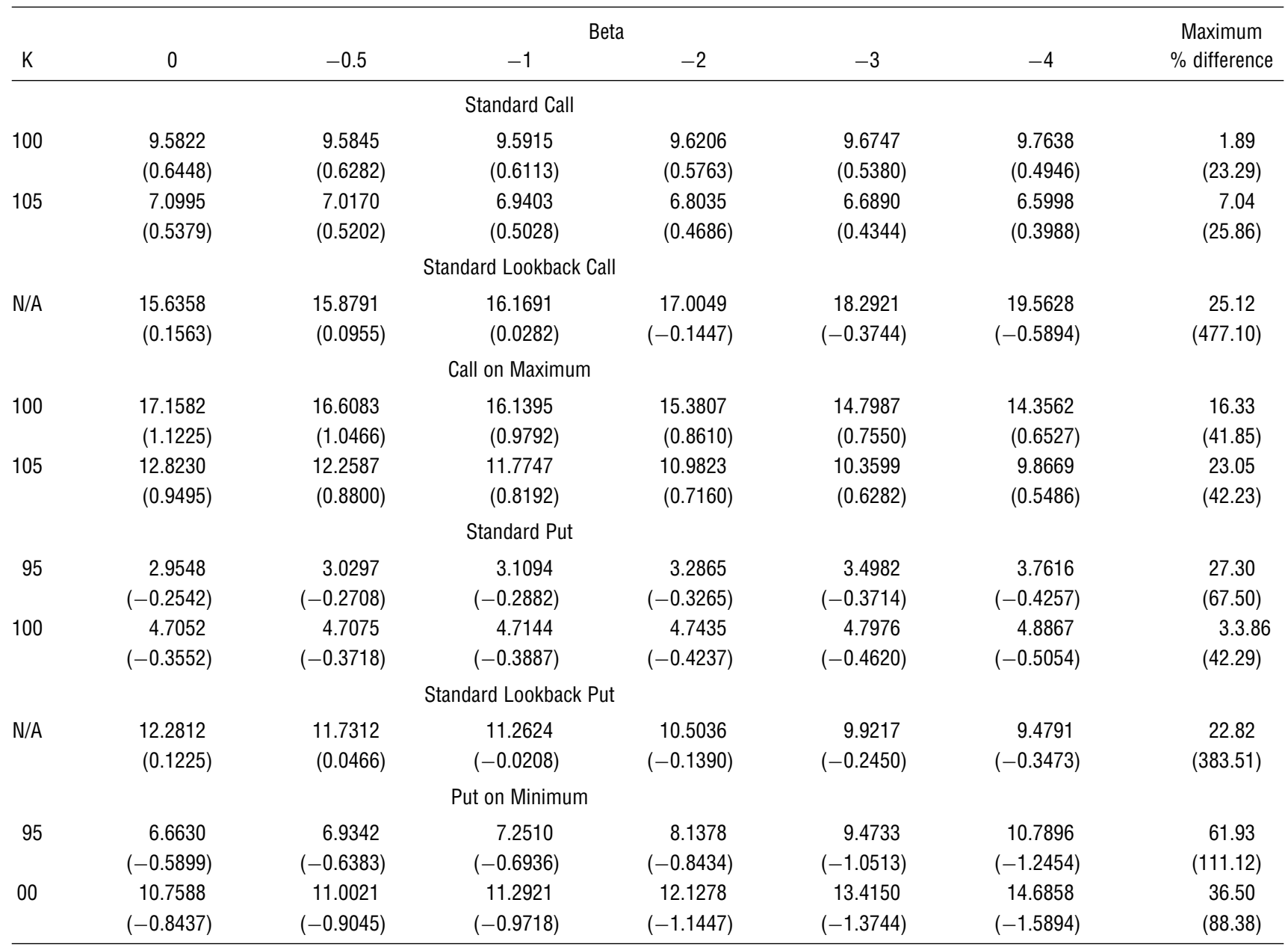

Note. Standard call, standard lookback call, call on maximum, standard put, standard lookback put and put on minimum prices and deltas under CEV processes with elasticities $\beta=0,-0.5,-1,-2,-3$, and -4 . The value of delta is given in parentheses underneath the corresponding option price. The utmost right column gives the absolute value of the percentage difference between the Black-Scholes price (delta) and CEV price (delta) with $\beta=-4$ relative to the Black-Scholes price (delta). The strike price $K$, varies as indicated in the left column. Parameters used in the calculation: $\sigma_{0}=\sigma(100)=0.25, r=0.1$, $q=0, T=0.5$.

Second, we apply the analytical formulae to carry out a comparative statics analysis and demonstrate that, in the presence of a CEV-based volatility smile, barrier and lookback option prices and their hedge ratios can deviate dramatically from the lognormal values. In particular, up-and-out, double knock-out, and lookback call prices and deltas are extremely sensitive to the specification of the elasticity parameter $\beta$. Strikingly, we show that their deltas can have different signs under lognormal and CEV specifications, as well as CEV specifications with different elasticities. Finally, we carry out a dynamic hedging simulation experiment to quantify the impact of model misspecification on the outcome of dynamic delta-hedging strategies. We find that it is much more important to have the accurate model specification for hedging barrier options that are canceled in-the-money than for standard options and barrier options that are canceled out-of-the-money. Notably, it is possible to do significantly worse by delta-hedging the former con- 
tracts with a misspecified model than by not hedging at all.

The results of this paper have important implications for financial institutions making markets in path-dependent options. These results make a strong case for moving beyond the simplistic geometric Brownian motion assumption to more realistic models incorporating volatility smiles.

\section{Acknowledgments}

The authors thank John Birge, Jim Bodurtha, Phelim Boyle, Peter Carr, Darrell Duffie, Dilip Madan, Eric Reiner, Mark Rubinstein, and Marc Yor for stimulating comments. The authors are especially grateful to Phelim Boyle for his support with this project.

\section{Appendix A. Proofs}

Proof of Proposition 1. In our characterization of the functions $\psi_{r}$ and $\phi_{r}$ we follow Borodin and Salminen (1996, pp. 18-19). Equation (3) is proved as follows. The function $u(S):=$ $\mathbf{E}_{S}\left[e^{-r J_{L}} \mathbf{1}_{\left\{\Phi_{L}<\Im_{U}\right\}}\right]$ satisfies the ODE (8) with the boundary conditions $u(L)=1$ and $u(U)=0$. Any solution of (8) can be expressed as a linear combination of the fundamental solutions $\psi_{r}$ and $\phi_{r}$. We look for $u(S)$ in the form $C_{1} \psi_{r}(S)+C_{2} \phi_{r}(S)$ with the boundary conditions $C_{1} \psi_{r}(L)+C_{2} \phi_{r}(L)=1$ and $C_{1} \psi_{r}(U)+C_{2} \phi_{r}(U)=0$. Solving for $C_{1}$ and $C_{2}$ yields Equation (3). Equation (2) is obtained by taking the limit $U \rightarrow \infty$ and using the boundary properties (12). Equations (4) and (5) are proved similarly. Finally, Equation (6) follows from the identity $e^{-r \Phi(L, U)}=e^{-r \Phi_{L}} \mathbf{1}_{\left\{\Phi_{L}<T_{U}\right\}}+e^{-r \Phi_{U}} \mathbf{1}_{\left\{\Phi_{U}<\Phi_{L}\right\}}$.

Proof of Proposition 2. By Fubini's theorem:

$$
\begin{aligned}
\int_{0}^{\infty} e^{-\lambda T} \mathbf{E}_{S}\left[\mathbf{1}_{\{\theta \leq T\}} e^{-r \theta}\right] d T & =\mathbf{E}_{S}\left[\int_{\theta}^{\infty} e^{-\lambda T} d T e^{-r \theta}\right] \\
& =\frac{1}{\lambda} \mathbf{E}_{S}\left[e^{-(r+\lambda) \theta}\right] .
\end{aligned}
$$

Proof of Proposition 3. Let $p(t ; S, Y)$ be the transition density of the diffusion $\left\{S_{t}, t \geq 0\right\}$ starting at $S_{0}=S$ with two absorbing barriers at $L$ and $U, 0<L<S<U<\infty$, and let $G_{\lambda}(S, Y)$ be the resolvent kernel (Green's function) defined as the Laplace transform of the transition density, $G_{\lambda}(S, Y):=\int_{0}^{\infty} e^{-\lambda t} p(t ; S, Y) d t, \lambda>0$. The Green's function with two absorbing barriers can be expressed in the form (see Borodin and Salminen 1996, p. 19; note that we work with the Green's function with respect to the Lebesgue measure, while Boroding and Salminen-with respect to the speed measure, and our Green's function (40) differs from Borodin and Salminen's by a factor $\mathrm{m}(Y))(a \wedge b:=\min \{a, b\}, a \vee b:=\max \{a, b\})$ :

$$
G_{\lambda}(S, Y)=\frac{\mathfrak{m}(Y)}{W_{\lambda}} \Psi_{\lambda}(S \wedge Y) \Phi_{\lambda}(S \vee Y),
$$

where the functions $\Psi_{\lambda}(S)$ and $\Phi_{\lambda}(S)$ can be characterized as the unique (up to a multiplicative factor) solutions of the ODE (8) by firstly demanding that $\Psi_{\lambda}(S)$ is increasing and $\Phi_{\lambda}(S)$ is decreasing on $[L, U]$, and secondly posing the boundary conditions $\Psi_{\lambda}(L)=0$ and $\Phi_{\lambda}(U)=0$. The $W_{\lambda}$ is the Wronskian of the functions $\Psi_{\lambda}$ and $\Phi_{\lambda}$ with respect to the scale density defined by: $\Phi_{\lambda}(S) \Psi_{\lambda}^{\prime}(S)-$ $\Psi_{\lambda}(S) \Phi_{\lambda}^{\prime}(S)=\mathfrak{G}(S) W_{\lambda}$ (the prime denotes differentiation with respect to $S$ ). The functions $\Psi_{\lambda}(S)$ and $\Phi_{\lambda}(S)$ can be uniquely (up to a multiplicative factor) expressed in terms of the fundamental solutions $\psi_{\lambda}(S)$ and $\phi_{\lambda}(S)$ of the problem without barriers: $\Psi_{\lambda}(S)=\Delta_{\lambda}(L, S)$ $\Phi_{\lambda}(S)=\Delta_{\lambda}(S, U)$, where $\Delta_{\lambda}(A, B)$ is defined in Equation (7). The Wronskian is: $W_{\lambda}=w_{\lambda} \Delta_{\lambda}(L, U)$, where $w_{\lambda}$ is the Wronskian of $\phi_{\lambda}$ and $\psi_{\lambda}$ (13). Substituting these expressions into Equation (40), we obtain a representation of the Green's function with two absorbing barriers in terms of the fundamental solutions $\psi_{\lambda}(S)$ and $\phi_{\lambda}(S)$ of the problem without barriers:

$$
G_{\lambda}(S, Y)=\frac{\mathfrak{m}(Y)}{w_{\lambda} \Delta_{\lambda}(L, U)} \Delta_{\lambda}(L, S \wedge Y) \Delta_{\lambda}(S \vee Y, U)
$$

To prove Equation (21), take the Laplace transform of the expectation in Equation (17) in time to expiration T. By Fubini's theorem:

$$
\begin{aligned}
\int_{0}^{\infty} e^{-\lambda T} \mathbf{E}_{S}\left[\mathbf{1}_{\left\{T_{(L, U)}>T\right\}}\left(S_{T}-K\right)^{+}\right] d T \\
\quad=\int_{0}^{\infty} e^{-\lambda T}\left(\int_{K}^{U}(Y-K) p(T ; S, Y) d Y\right) d T \\
\quad=\int_{K}^{U}(Y-K)\left(\int_{0}^{\infty} e^{-\lambda T} p(T ; S, Y) d T\right) d Y \\
=\int_{K}^{U}(Y-K) G_{\lambda}(S, Y) d Y .
\end{aligned}
$$

Substituting the expression (41) for the resolvent into the last integral yields Equation (21). Note that the integrals (19) and (20) always exist because $\psi_{\lambda}$ and $\phi_{\lambda}$ are continuous on any closed interval $[A, B], 0<A<B<\infty$.

Proof of Lemma 1. Equation (22) follows from the identity $\mathbf{Q}_{x}\left(m_{t} \leq y\right)=\mathbf{Q}_{x}\left(\mathscr{T}_{y} \leq t\right)$ for $y \leq x$, where $\mathscr{T}_{y}$ is the first hitting time of $y$, Equation (2), and Equation (16) in the limit $r \rightarrow 0$. Equation (23) follows from the identity $\mathbf{Q}_{x}\left(M_{t} \geq y\right)=\mathbf{Q}_{x}\left(\mathscr{T}_{y} \leq t\right)$ for $y \geq x$, Equation (4), and Equation (16) in the limit $r \rightarrow 0$.

Proof of Proposition 4. To prove Equation (27), note that $\left(K-m_{T}\right)^{+}=\int_{0}^{K} \mathbf{1}_{\left\{m_{T} \leq y\right\}} d y$, and $m_{T}=\min \left\{m_{t}, m_{t, T}\right\}$, where $m_{t, T}=\min _{t \leq u \leq T} S_{u}$. Then by the Markov property and time homogeneity:

$$
\begin{aligned}
\mathbf{E}_{t}\left[\left(K-m_{T}\right)^{+}\right] & =\int_{0}^{K} \mathbf{Q}\left(m_{T} \leq y \mid \mathscr{F}_{t}\right) d y \\
& = \begin{cases}\int_{0}^{K} F\left(y ; S_{t}, \tau\right) d y, & m_{t} \geq K \\
K-m_{t}+\int_{0}^{m_{t}} F\left(y ; S_{t}, \tau\right) d y, & m_{t}<K .\end{cases}
\end{aligned}
$$

To prove Equation (24), note that $m_{T} \leq S_{T}, m_{T} \leq m_{t}$, and $\mathbf{E}_{t}\left[S_{T}\right]=$ $e^{(r-q) \tau} S_{t}$. Then,

$$
\begin{aligned}
\mathbf{E}_{t}\left[\left(S_{T}-m_{T}\right)^{+}\right] & =\mathbf{E}_{t}\left[\left(S_{T}-m_{t}\right)\right]+\mathbf{E}_{t}\left[\left(m_{t}-m_{T}\right)\right] \\
& =e^{(r-q) \tau} S_{t}-m_{t}+\int_{0}^{m_{t}} F\left(y ; S_{t}, \tau\right) d y,
\end{aligned}
$$

where we used the result (27) with $K=m_{t}$. Equations (26) and (25) are proved similarly. 
Proof of Proposition 5. First consider the case with drift $\mu \neq 0$. We look for solutions in the form $u(S)=S^{\frac{1}{2}+\beta} e^{\frac{\epsilon}{2} x} w(x)$ for some unknown function $w(x)$ with $x$ and $\epsilon$ defined in Equations (34) and (35). Substituting this functional form for $u$ into Equation (36), we arrive at the ODE for $w$

$$
\frac{d^{2} w}{d x^{2}}+\left(-\frac{1}{4}+\frac{k}{x}+\frac{\frac{1}{4}-m^{2}}{x^{2}}\right) w=0, \quad x \in(0, \infty)
$$

This is the Whittaker's form of the confluent hypergeometric equation with two linearly independent solutions $M_{k, m}(x)$ and $W_{k, m}(x)$ (see Abramowitz and Stegun (1972, p. 505) and Slater 1960). Now we need to verify boundary properties of solutions (37) and (38). First, consider the case $\beta<0$. The CEV process has a natural boundary at infinity, an exit boundary at zero for $-1 / 2 \leq \beta<0$, and a regular boundary suplemented with the killing boundary condition for $\beta<-1 / 2$. The behavior at the right natural boundary is prescribed by Equation (12). The behavior at the left exit boundary is prescribed by Equation (10). At the killing boundary the boundary condition is (9). These boundary properties for solutions (37) and (38) are verified using the asymptotic properties of the Whittaker functions $M_{k, m}(x)$ and $W_{k, m}(x)$ as $x \rightarrow 0$ and $x \rightarrow \infty$ (see Slater 1960).

Next consider the case $\beta>0$. The CEV process has an entrance boundary at infinity and a natural boundary at zero. The behavior at the left natural boundary is prescribed by Equation (11), and at the right entrance boundary-by (Borodin and Salminen 1996, pp. 18-19): $\lim _{S \rightarrow \infty} \psi_{\lambda}(S)=+\infty, \lim _{S \rightarrow \infty} \phi_{\lambda}(S)>0$. These boundary properties are verified using the asymptotic properties of the Whittaker functions (note that for $\beta>0$ the change of variable $x=$ $\left(|\mu| / \delta^{2}|\beta|\right) S^{-2 \beta}$ maps the origin onto infinity, and infinity onto the origin)

The Wronskian of the Whittaker equation is (Slater 1960, p. 26; the prime denotes differentiation in $x): W_{k, m}(x) M_{k, m}^{\prime}(x)-$ $M_{k, m}(x) W_{k, m}^{\prime}(x)=\Gamma(2 m+1) / \Gamma\left(m-k+\frac{1}{2}\right)$, leading to the CEV Wronskian (39) for $\mu \neq 0$.

Next consider the case without drift, $\mu=0$. We look for solution in the form $u(S)=S^{\frac{1}{2}} v(\sqrt{2 \lambda} z)$ for some unknown function $v(z)$ with $z$ defined in Equation (34). Substituting this functional form into Equation (36) with $\mu=0$, we arrive at the ODE for $v$

$$
z^{2} \frac{d^{2} v}{d z^{2}}+z \frac{d v}{d z}-\left(2 \lambda z^{2}+v^{2}\right) v=0
$$

This is the modified Bessel equation with two linearly independent solutions $I_{\nu}(\sqrt{2 \lambda} z)$ and $K_{\nu}(\sqrt{2 \lambda} z)$ (Abramowitz and Stegun 1972 , p. 374). The boundary properties of solutions (37) and (38) are verified using the asymptotic properties of the modified Bessel functions (Abramowitz and Stegun 1972, pp. 375-378). The Wronskian of the modified Bessel functions is (Abramowitz and Stegun 1972, p. 375; the prime denotes differentiation in $z$ ) $K_{\nu}(z) I_{\nu}^{\prime}(z)-I_{\nu}(z) K_{\nu}^{\prime}(z)=1 / z$, leading to the CEV Wronskian (39) for $\mu=0$.

\section{Appendix B. Emanuel and MacBeth's (1982) Paradox for $\beta>0$}

For $\beta>0$, the CEV local volatility $\sigma(S)=\delta S^{\beta}$ is unbounded as $S \rightarrow \infty$, and $+\infty$ is an entrance boundary for the diffusion. This means that there exists a $\operatorname{limit}_{\lim _{S \rightarrow \infty}} p\left(T ; S, S_{T}\right)=p\left(T ; \infty, S_{T}\right)$ for the transition density, and the process can be started at infinity. If started at infinity, the process rapidly enters the state space and reaches any interior point before time $T>0$ with positive probability (see Karlin and Taylor 1981 and Borodin and Salminen 1996). Furthermore, Emanuel and MacBeth (1982) were the first to observe that the mean of the CEV density (31) for $\beta>0$ is less than $e^{\mu T} S$, as one would expect given the CEV equation of motion (28). For $\beta>0$, integrating $S_{T}$ with the density one obtains $\mathbf{E}_{S}\left[S_{T}\right]=e^{\mu T} S(1-$ $G(\nu, \zeta / 2))$, where $G(\nu, x)$ is the complementary Gamma distribution function and $\zeta$ is defined in Equation (33). Thus, the mean of $S_{T}$ is less than $e^{\mu T} S$ for all $S>0$ and $T>0$. Therefore, the process $e^{-\mu t} S_{t}$ is a strict local martingale and a strict super-martingale on the time interval [0, T] (see Elworthy et al. 1999). Consequently, there is no equivalent martingale measure for this specification of local volatility (see Sin 1996). From the practical point of view, this problem can be easily avoided. Pick a large fixed number $\mathscr{E}$ and modify the volatility specification: $\sigma_{\mathscr{E}}(S)=\delta \min \left\{S^{\beta}, \mathscr{E}^{\beta}\right\}$. This modification is termed limited CEV (LCEV) process by Andersen and Andreasen (1998). Roughly speaking, when the asset price crosses over the "switching level" \& $\mathscr{E}$, the LCEV process becomes a geometric Brownian motion. The LCEV volatility is bounded, $+\infty$ is a natural boundary, the mean of $S_{T}$ is equal to $e^{\mu T} S$, and the process $\left\{e^{-\mu t} S_{t} ; t \geq 0\right\}$ is a martingale on any finite time interval. A similar regularization can be applied at small price levels for the process with $\beta<0$ to avoid absorption at zero.

\section{Appendix C. Integrals $I_{\lambda}$ and $J_{\lambda}$ for the CEV Process}

First consider the case with $\mu \neq 0$. The speed density (18) for the CEV process with $\mu \neq 0$ is $\mathrm{m}(Y)=2 \delta^{-2} Y^{-2 \beta-2} e^{-\epsilon y}$. Using the indefinite integrals reported by Slater (1960), pp. 23-25, the integrals in Equations (19) and (20) for the CEV fundamental solutions (37) and (38) are calculated in closed form $\left(y:=\frac{|\mu|}{\delta^{2}|\beta|} Y^{-2 \beta}, C:=\delta \sqrt{|\beta \mu|}\right)$ :

$C I_{\lambda}(K, A, B)= \begin{cases}\left(\frac{Y^{\frac{1}{2}}}{2 m+1} e^{\frac{y}{2}} M_{k+\frac{1}{2}, m+\frac{1}{2}}(y)\right. & \\ \left.-\frac{2 m K Y^{-\frac{1}{2}}}{m-k-\frac{1}{2}} e^{\frac{y}{2}} M_{k+\frac{1}{2}, m-\frac{1}{2}}(y)\right)\left.\right|_{Y=A^{\prime}} ^{Y=B} & \beta<0, \mu>0, \\ \left(\frac{Y^{\frac{1}{2}}}{2 m+1} e^{-\frac{y}{2}} M_{k-\frac{1}{2}, m+\frac{1}{2}}(y)\right. & \\ \left.+\frac{2 m K Y^{-\frac{1}{2}}}{m+k-\frac{1}{2}} e^{-\frac{y}{2}} M_{k-\frac{1}{2}, m-\frac{1}{2}}(y)\right)\left.\right|_{Y=A^{\prime}} ^{\gamma=B} & \beta<0, \mu<0, \\ \left(Y^{\frac{1}{2}} e^{-\frac{y}{2}} W_{k-\frac{1}{2}, m-\frac{1}{2}}(y)\right. & \\ \left.+K Y^{-\frac{1}{2}} e^{-\frac{y}{2}} W_{k-\frac{1}{2}, m+\frac{1}{2}}(y)\right)\left.\right|_{Y=A^{\prime}}, & \beta>0, \mu>0, \\ \left(\frac{Y^{\frac{1}{2}}}{k-m+\frac{1}{2}} e^{\frac{y}{2}} W_{k+\frac{1}{2}, m-\frac{1}{2}}(y)\right. & \\ \left.-\frac{K Y^{-\frac{1}{2}}}{k+m+\frac{1}{2}} e^{\frac{y}{2}} W_{k+\frac{1}{2}, m+\frac{1}{2}}(y)\right)\left.\right|_{Y=A^{\prime}} ^{Y=B} & \beta>0, \mu<0,\end{cases}$ 


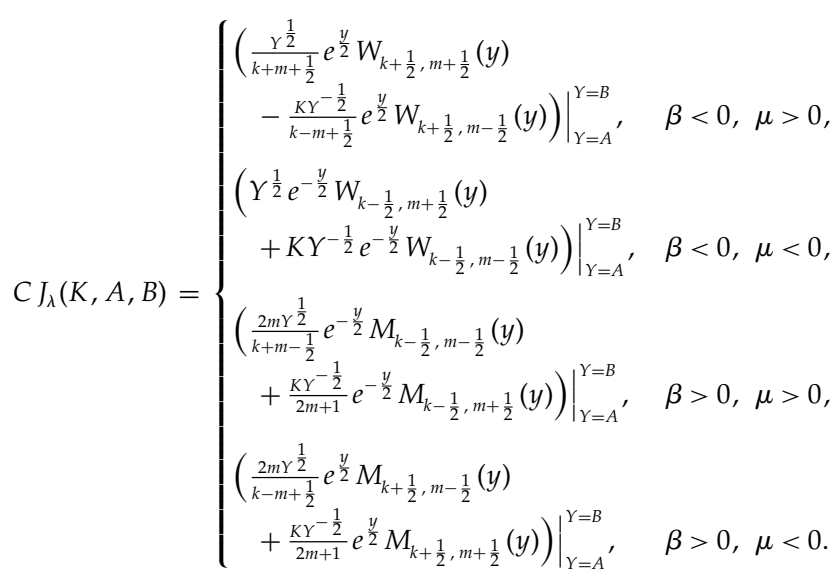

Next consider the case with $\mu=0$. The speed density for the CEV process with $\mu=0$ is $\mathfrak{m}(Y)=2 \delta^{-2} Y^{-2 \beta-2}$. Using the integrals reported by Prudnikov et al. 1986, p. 39, the integrals in Equations (19) and (20) are calculated in closed form $\left(y:=\frac{1}{\delta|\beta|} Y^{-\beta}\right)$ :

$$
\begin{aligned}
& I_{\lambda}(K, A, B)=\frac{2}{\delta \sqrt{2 \lambda}}\left\{\begin{array}{cc}
\left(Y^{\frac{1}{2}-\beta} I_{\nu+1}(\sqrt{2 \lambda} y)\right. \\
\left.-K Y^{-\frac{1}{2}-\beta} I_{\nu-1}(\sqrt{2 \lambda} y)\right)\left.\right|_{Y=A} ^{Y=B}, & \beta<0, \\
Y^{\frac{1}{2}-\beta} K_{\nu+1}(\sqrt{2 \lambda} y) \\
\left.-K Y^{-\frac{1}{2}-\beta} K_{\nu-1}(\sqrt{2 \lambda} y)\right)\left.\right|_{Y=A} ^{Y=B}, & \beta>0,
\end{array}\right. \\
& J_{\lambda}(K, A, B)=\frac{2}{\delta \sqrt{2 \lambda}}\left\{\begin{array}{cc}
\left(-Y^{\frac{1}{2}-\beta} K_{\nu+1}(\sqrt{2 \lambda} y)\right. \\
\left.+K Y^{-\frac{1}{2}-\beta} K_{\nu-1}(\sqrt{2 \lambda} y)\right)\left.\right|_{Y=A} ^{Y=B}, & \beta<0, \\
\left(-Y^{\frac{1}{2}-\beta} I_{\nu+1}(\sqrt{2 \lambda} y)\right. \\
\left.+K Y^{-\frac{1}{2}-\beta} I_{\nu-1}(\sqrt{2 \lambda} y)\right)\left.\right|_{Y=A} ^{Y=B}, & \beta>0 .
\end{array}\right.
\end{aligned}
$$

\section{Appendix D. Laplace Transform Inversion}

To compute barrier and lookback option prices we need to invert Laplace transforms in Equations (16), (21)-(23). Equations (16), (21)-(23) have the form $\int_{0}^{\infty} e^{-\lambda t} f(t) d t=F(\lambda)$, where $\lambda>0, F(\lambda)$ is the known Laplace transform, and $f(t)$ is the original function to be determined. All originals in Equations (16), (21)-(23) are continuous and bounded on $[0, \infty)$. Then the Laplace transform converges for all complex $\lambda$ with $\operatorname{Re}(\lambda)>0, F(\lambda)$ is a single-valued analytic function of $\lambda$ in the half-plane $\operatorname{Re}(\lambda)>0$, and the inverse Laplace transform can be calculated as the Bromwich contour integral along the line $\operatorname{Re}(\lambda)=c>0$. To compute the integral, we use the Euler numerical integration algorithm due to Abate and Whitt (1995). This algorithm was applied to option pricing problems by Fu, Madan, and Wang (1997) and Davydov and Linetsky (2000a). Our implementation follows Appendix B in Davydov and Linetsky (2000a). We found this algorithm very accurate for all computations in this paper. Alternatively, the Laplace transforms for barrier and lookback options under the CEV process can be inverted analytically, and the results are expressed as eigenfunction expansions for some Sturm-Liouville problems associated with the CEV process (Davydov and Linetsky 2000b).

\section{References}

Abate, J., W. Whitt. 1995. Numerical inversion of laplace transforms of probability distributions. ORSA J. Comput. 7 36-43.

Abramowitz, M., I. A. Stegun. 1972. Handbook of Mathematical Functions. Dover, NY.

Andersen, L., J. Andreasen. 1998. Volatility skews and extensions of the LIBOR market model. Working paper, General Re, New York.

Black, F., M. Scholes. 1973. The pricing of options and corporate liabilities. J. Political Econom. 81 637-659.

Borodin, A. N., P. Salminen. 1996. Handbook of Brownian Motion. Birkhauser, Boston, MA.

Boyle, P. P., Y. Tian. 1999. Pricing lookback and barrier options under the CEV process. J. Financial and Quant. Anal. 34 (Correction: P. P. Boyle, Y. Tian, J. Imai. Lookback options under the CEV process: A correction. JFQA web site at $/$ http://depts. washington.edu/jfqa/> in Notes, comments, and corrections).

Broadie, M., J. Detemple. 1995. American capped call options on dividend-paying assets. Rev. Financial Stud. 8 161-191.

Carr, P. 1998. Randomization and the American put. Rev. Financial Stud. 11 597-626.

, J. F. Picron. 1999. Static hedging of timing risk. J. Derivatives Spring 57-70.

Conze, A., R. Vishwanathan. 1991. Path-dependent options: The case of lookback options. J. Finance 46 1893-1907.

Cox, J. 1975. Notes on option pricing I: Constant elasticity of variance diffusions. Working paper, Stanford University (reprinted in Journal of Portfolio Management, 1996, 22 15-17).

, S. Ross. 1976. The valuation of options for alternative stochastic processes. J. Financial Econom. 3 145-166.

Davydov, D., V. Linetsky. 2000a. Structuring, pricing and hedging double-barrier step options. Forthcoming in Journal of Computational Finance.

2000b. Pricing options on scalar diffusions: An eigenfunction expansion approach. Working paper, Northwestern University.

Derman, E., I. Kani. 1996. The ins and outs of barrier options. Derivatives Quart. Winter 96 (Part 1) and Spring 97 (Part 2).

Duffie, D. 1996. Dynamic Asset Pricing, 2nd ed. Princeton University Press, Princeton N.J.

Eie, B. 1983. The generalized bessel process corresponding to an ornstein-Uhlenbeck process. Scandinavian J. Statist. 10 247-250.

Elworthy, K. D., X.-M. Li, M. Yor. 1999. The importance of strictly local martingales: Applications to radial Ornstein-Uhlenbeck processes. Probability Theory and Related Fields 115, 325-355.

Emanuel, D., J. MacBeth. 1982. Further results on the constant elasticity of variance call option pricing model. J. Financial and Quant. Anal. 17 533-554. 
Feller, W. 1951. Two singular diffusion problems. Ann. Math. 54 173-182.

Fu, M., D. Madan, T. Wang. 1997. Pricing Asian options: A comparison of analytical and Monte Carlo methods. J. Comput. Finance 2 49-74.

Geman, H., M. Yor. 1993. Bessel processes, Asian options, and perpetuities. Math. Finance 3 349-375.

_ 1 . 1995. Pricing and hedging double barrier options: A probabilistic approach. Math. Finance 6 365-378.

Giorno, V., A. G. Nobile, L.M. Ricciardi, L. Sacerdote. 1986. Some remarks on the Rayleigh process. J. Appl. Probab. 23 398-408.

Going-Jaeschke, A., M. Yor. 1999. A survey and some generalizations of Bessel processes. Preprint, Laboratoire de Probabilites, Universites de Paris VI and VII.

Goldenberg, D. 1991. A unified method for pricing options on diffusion processes. J. Financial Econom. 29 3-34.

Goldman, M. B., H. B. Sosin, M. A. Gatto. 1979. Path-dependent options. J. Finance 34 1111-1127.

- - L. Shepp. 1979. On contingent claims that insure ex-post optimal stock market timing. J. Finance 34 401-413.

Ingersoll, J. E. 1987. Theory of Financial Decision Making. Rowman and Littlefield, Savage, MD.

Jackwerth, J. C., M. Rubinstein. 1998. Recovering stochastic processes from option prices. Working paper, University of California, Berkeley, CA.

Karatzas, I., S. Shreve. 1991. Brownian Motion and Stochastic Calculus, 2nd ed. Springer-Verlag, New York.

Karlin, S., H. M. Taylor. 1981. A Second Course in Stochastic Processes. Academic Press, San Diego, CA.
Kunitomo, N., M. Ikeda. 1992. Pricing options with curved boundaries. Math. Finance 2 275-298.

Linetsky, V. 1999. Step options. Math. Finance, 9 55-96.

Merton, R. C. 1973. Theory of rational options pricing. Bell J. Econom. Management Sci. 4 141-183.

Pelsser, A. 2000. Pricing double barrier options using analytical inversion of Laplace transforms. Finance and Stochastics 4 95-104.

Prudnikov, A. P., Yu. A. Brychkov, O. I. Marichev. 1986. Integrals and Series Vol. 2, Gordon and Breach, New York.

Reiner, E. 1994. In search of parsimony: Valuing standard and pathdependent options for alternative stochastic processes. Derivatives conf. Toronto, Canada.

Revuz, D., M. Yor. 1999. Continuous Martingales and Brownian Motion, 3rd ed. Springer, Berlin, Germany.

Rubinstein, M., E. Reiner. 1991. Breaking down the barriers. RISK 4 (Sept.) 28-35.

Schroder, M. 1989. Computing the constant elasticity of variance option pricing formula. J. Finance 44 211-219.

Schroder, M. 2000. On the valuation of double-barrier options: Computational aspects. Forthcoming in J. Comput. Finance.

Sin, C. 1996. Strictly local martingales and hedge ratios on stochastic volatility models. Ph.D. thesis, Cornell University, New York.

Shiga, T., S. Watanabe. 1973. Bessel diffusions as a one-parameter family of diffusion processes. Z. Wahrscheinlichkeitstheorie Verw. Geb. 27 37-46.

Slater, L. J. 1960. Confluent Hypergeometric Functions, Cambridge University Press, Cambridge, U.K.

Snyder, G. L. 1969. Alternative forms of options. Financial Analysts J. 25 93-99.

Accepted by Phelim P. Boyle; received August 2000. This paper was with the authors 2 months for 1 revision. 